\title{
An fMRI study of affective perspective taking in individuals with psychopathy: imagining another in pain does not evoke empathy
}

\author{
Jean Decety ${ }^{1,2 *}$, Chenyi Chen ${ }^{1}$, Carla Harenski ${ }^{3,4}$ and Kent A. Kieh/ ${ }^{3,4}$ \\ ${ }^{1}$ Department of Psychology, University of Chicago, Chicago, IL, USA \\ ${ }^{2}$ Department of Psychiatry and Behavioral Neuroscience, University of Chicago, Chicago, IL, USA \\ ${ }^{3}$ Departments of Psychology and Neuroscience, University of New Mexico, Albuquerque, NM, USA \\ ${ }^{4}$ Mind Research Network, Albuquerque, NM, USA
}

\section{Edited by:}

Josef Parvizi, Stanford University, USA

\section{Reviewed by:}

Lucina Q. Uddin, Stanford

University, USA

Ezequiel Gleichgerrcht, Favaloro

University, Argentina

\section{${ }^{*}$ Correspondence:}

Jean Decety, Department of

Psychology, Department of

Psychiatry and Behavioral

Neuroscience, University of

Chicago, 5848 S. University Avenue,

Chicago, IL 60637, USA

e-mail:decety@uchicago.edu
While it is well established that individuals with psychopathy have a marked deficit in affective arousal, emotional empathy, and caring for the well-being of others, the extent to which perspective taking can elicit an emotional response has not yet been studied despite its potential application in rehabilitation. In healthy individuals, affective perspective taking has proven to be an effective means to elicit empathy and concern for others. To examine neural responses in individuals who vary in psychopathy during affective perspective taking, 121 incarcerated males, classified as high $(n=37$; Hare psychopathy checklist-revised, PCL-R $\geq 30$ ), intermediate $(n=44 ; \mathrm{PCL}-\mathrm{R}$ between 21 and $29)$, and low $(n=40 ; P C L-R \leq 20)$ psychopaths, were scanned while viewing stimuli depicting bodily injuries and adopting an imagine-self and an imagine-other perspective. During the imagine-self perspective, participants with high psychopathy showed a typical response within the network involved in empathy for pain, including the anterior insula (alNS), anterior midcingulate cortex (aMCC), supplementary motor area (SMA), inferior frontal gyrus (IFG), somatosensory cortex, and right amygdala. Conversely, during the imagine-other perspective, psychopaths exhibited an atypical pattern of brain activation and effective connectivity seeded in the anterior insula and amygdala with the orbitofrontal cortex (OFC) and ventromedial prefrontal cortex (vmPFC). The response in the amygdala and insula was inversely correlated with PCL-R Factor 1 (interpersonal/affective) during the imagine-other perspective. In high psychopaths, scores on PCL-R Factor 1 predicted the neural response in ventral striatum when imagining others in pain. These patterns of brain activation and effective connectivity associated with differential perspective-taking provide a better understanding of empathy dysfunction in psychopathy, and have the potential to inform intervention programs for this complex clinical problem.

Keywords: amygdala, effective connectivity, empathy, insula, orbitofrontal cortex, perspective taking, psychopathy, ventral striatum
Empathy, the social-emotional response that is induced by the perception of another person's affective state, is a fundamental component of emotional experience, and plays a vital role in social interaction (Szalavitz and Perry, 2010). It is thought to be a proxy for prosocial behavior, guiding our social preferences and providing the affective and motivational base for moral development. Empathy is a deeply fundamental component of healthy co-existence whose absence is the hallmark of serious social-cognitive dysfunctions. Among the various psychopathologies marked by such deficits, psychopaths are characterized by a general lack of empathy and attenuated responding to emotional stimuli (Blair et al., 1997; Herpertz and Sass, 2000; Hare, 2003; Mahmut et al., 2008).

Empathy includes both cognitive and affective components (Decety and Jackson, 2004; Shamay-Tsoory, 2009; Singer and Lamm, 2009; Decety, 2011a; Zaki and Ochsner, 2012).
The empathic arousal component, or emotion contagion, develops earlier than the cognitive component, and seems to be hardwired in the brain with deep evolutionary roots (Decety and Svetlova, 2012). In addition developmental research has found that concern for others emerges prior to the second year of life. In these studies, young children are not only moved by others' emotional states, but they make distress and pain attribution in conjunction with their comforting behavior and recognize what the target is distressed about (Roth-Hanania et al., 2011). Empathic arousal plays a fundamental role in generating the motivation to care and help another person in distress and depends only minimally on mindreading and perspective-taking capacities. In naturalistic studies, young children with high empathy disposition are more readily aroused vicariously by other' sadness, pain or distress, but at the same time possess greater capacities for emotion regulation so that their own negative arousal motivates 
rather than overwhelms their desire to alleviate the other's distress (Miller and Jansen op de Haar, 1997; Nichols et al., 2009). Empathic arousal is a bottom-up process in which the amygdala, hypothalamus, anterior insula (aINS), and orbitofrontal cortex (OFC) underlie rapid and prioritized processing of emotion signals sent by others (Decety and Svetlova, 2012). The cognitive component of empathy overlaps with the construct of perspective taking (Ruby and Decety, 2003). Perspective taking describes the ability to consciously put oneself into the mind of another individual and imagine what that person is thinking or feeling. The ability to adopt the perspective of another has previously been linked to social competence and social reasoning (Underwood and Moore, 1982). A substantial body of behavioral studies has documented that affective perspective taking is a powerful way to elicit empathy and concern for others (Batson et al., 1997; Decety and Hodges, 2006; Van Lange, 2008). For instance, Oswald (1996) found that affective perspective taking is more effective that cognitive perspective taking to evoke empathy and altruistic helping. Functional neuroimaging studies have consistently identified a circumscribed neural network reliably involved in perspective taking, which links the medial prefrontal cortex (mPFC), posterior superior temporal sulcus (pSTS/TPJ), and temporal poles/amygdala (Ruby and Decety, 2003, 2004; Hynes et al., 2006; Lawrence et al., 2006; Vollm et al., 2006; Rameson et al., 2011). Lesion studies have shown that affective perspective taking depends on intact medial and ventromedial prefrontal cortex (vmPFC) as well as regions in the posterior temporo-parietal cortex (Rankin et al., 2006). Importantly, neurological patients with damage to the vmPFC are found to exhibit a specific impairment in affective theory of mind tasks, sparing their cognitive empathy ability (Shamay-Tsoory et al., 2006).

In the empathy literature, a number of behavioral studies have documented a distinction between an imagine-self perspective and an imagine-other perspective (Batson, 2011). When adopting the former perspective, the central figure is oneself and one's own thoughts and feelings, and increases the salience of selfattributes. The imagining-other perspective involves an empathic attentional set in which the individual opens himself or herself in a deeply responsive way to the other person (Barrett-Lennard, 1981; Batson, 2009; Halpern, 2012). This distinction between imagine-self and imagine-other perspectives is also supported by functional neuroimaging research. For instance, when participants are asked to imagine being in physical pain themselves, they report greater pain intensity ratings and have greater activation in the aINS, aMCC, thalamus, and somatosensory cortex compared to imagining the same pain happening to another person (Jackson et al., 2006). The reverse contrast, imagining-other in pain vs. imagining oneself in pain, was associated with increased activity in the right pSTS and mPFC. Another study reported that self-perspective compared to other-perspective, when watching videos depicting facial expression of pain, led to higher activity in brain areas involved in the affective response to threat or pain, such as the amygdala, the insula, and the aMCC, as well as higher subjective ratings of personal distress (Lamm et al., 2007).

It is well established that individuals with psychopathy have limited aversive arousal to the distress and sadness cues of others (Van Honk and Schutter, 2006; Blair, 2007; Anderson and
Kiehl, 2011), but spared theory of mind and cognitive perspective taking capacities (Blair, 2005; but see Brook and Kosson, 2012). However, it is not known if, when they adopt the affective perspective taking of another person, the extent to which the active contemplation of another's affective experience modulates brain circuits involved in affective processing.

Building on past research on perspective taking and empathy with healthy participants (Jackson et al., 2006; Lamm et al., 2007; Decety and Porges, 2011) as well as a recent study of pain empathy in criminal psychopaths (Decety et al., 2013), incarcerated offenders with different levels of psychopathy on Factors 1 and 2 underwent fMRI scanning while watching visual stimuli depicting physical pain. To elicit first- or third-person perspective taking (or imagine-self and imagine-other perspectives respectively) we explicitly manipulated the task instructions given to the participants in the scanner before each block, by asking them to think of the situations as either occurring to them or to someone else. Factor 1 describes a constellation of affective and interpersonal traits considered to be fundamental to the construct of psychopathy, which includes shallow affect, callous and lack of empathy, while Factor 2 reflects an unstable and antisocial lifestyle (Hare, 2003). Based on fMRI studies that used similar instructions and stimuli with healthy participants, it was predicted that imagine-self perspective would be associated with stronger visceromotor response in the aINS, somatosensory cortex and ACC than imagine-other perspective taking in participants scoring low on the psychopathy checklist-revised (PCL-R), especially Factor 1 , because these regions have been associated with activation of representations of pain and of other negative emotions (Benuzzi et al., 2008). However, due to altered responding to affective stimuli in psychopathy, the opposite effect was expected for individuals scoring high on psychopathy PCL-R Factor 1. When instructed to adopt the perspective of another individual in physical pain, we hypothesized that individuals scoring high on the PCL-R would show a pronounced deficit in aINS and vmPFC hemodynamic response. This prediction is based on the large body of evidence from lesion studies and neuroimaging studies with healthy individuals as well as with psychopaths that show the importance of these regions in affective perspective taking and empathic concern (Rankin et al., 2003; Shamay-Tsoory et al., 2003; Kiehl, 2006; Gleichgerrcht et al., 2011; Rameson et al., 2011; Decety et al., 2012; Young and Dungan, 2012). The distinction between imagine-self and imagine-other is critical, as most studies suggest that psychopaths have spared mentalizing (cognitive empathy) abilities, and that the key deficit appears to relate to their lack of concern about the impact of their behavior on potential victims, rather than the inability to adopt a victim-centered perspective (Dolan and Fullam, 2004).

Finally, analyses of functional segregation can be complemented by effective connectivity analyses. Whereas standard contrast analyses create a "snapshot" of regional brain activity in response to a task or condition, functional connectivity analyses can identify patterns of communication between regions that contrast analyses may not detect [see Decety and Porges, 2011; Zaki et al. (2007) for such methods in empathy for pain]. Given the role of the insula in mapping internal states of bodily and subjective feelings (Craig, 2002) and that of the amygdala 
in motivational salience (Cunningham and Brosch, 2012), these two regions were selected as seeds for the functional connectivity analyses.

\section{MATERIALS AND METHODS PARTICIPANTS}

One hundred twenty-four adult right-handed males between the ages of 18 and 50, incarcerated in a medium-security North American correctional facility, volunteered for the study and provided informed consent to the procedures described here, which were approved by the Institutional Review Boards of the University of New Mexico and the University of Chicago. Participants underwent the PCL-R assessment, including file review and interview, conducted by trained research assistants under the supervision of Dr. Kiehl. Three participants were excluded for excessive movement in the scanner. Participants scoring 30 and above on the PCL-R were assigned to the highpsychopathy group ( $n=37$; age $32.5 \pm 7.8$; IQ $103.3 \pm 13$ ). To create the medium- and low-psychopathy groups, two groups of volunteers were matched to high scorers on age, race and ethnicity, IQ (WAIS), comorbidity for DSM-IV Axis II disorders, and past drug abuse and dependence, from pools of incarcerated volunteers scoring between 21 and $29(n=44$; age $34.1 \pm 7$; IQ $97.3 \pm 12.7)$, and volunteers scoring below 20 on the PCL-R $(n=40$; age $34.6 \pm 6.9$; IQ $99.3 \pm 14)$, respectively. Participants were paid for their participation in the study.

\section{EXCLUSION CRITERIA}

Additional participants who volunteered for the study but met exclusion criteria were not included. Exclusion criteria were age younger than 18 years or older than 55, non-fluency in English, reading level lower than 4 th grade, IQ score lower than 80 , history of seizures, prior head injury with loss of consciousness $>30 \mathrm{~min}$, current Diagnostic and Statistical Manual of Mental Disorders (4th ed.; American Psychiatric Association, 1994) Axis I diagnosis, lifetime history of a psychotic disorder or psychotic disorder in a first degree relative, or current alcohol or drug use.

\section{TASK DESIGN}

Participants in the MRI scanner were instructed to adopt either a self-perspective or an other-perspective while viewing visual stimuli depicting right hands and right feet of individuals in painful and non-painful situations [stimuli and procedure similar to Jackson et al. (2006)]. All stimuli showed familiar events that can happen in everyday life to people (e.g., pinching one's finger in a door, or catching one's toe under a heavy object). Various types (mechanical, thermal and pressure) of pain inflicted to the limbs were depicted. Neutral pictures showed limbs in visually similar situations without pain component (e.g., a hand on the handle of a drawer as opposed to being caught in the same drawer). Participants viewed 120 stimuli of pain and no pain. Each trial lasted $1.4 \mathrm{~s}$ and consisted of one of the pain scenarios, and the inter-stimuli intervals were jittered between 2.5 and $5.4 \mathrm{~s}$. Timing parameters were generated using a genetic optimization algorithm (Wager and Nichols, 2003). Eye-tracking was monitored in the scanner to ensure that participants were paying attention to the stimuli.

\section{PERSPECTIVE INSTRUCTIONS}

A mixed block-event related fMRI design [24 blocks (12 imagineself and 12 imagine-other) with a total 120 trials] was employed, in which instructions were given to the subjects at the beginning of each block, i.e., for the imagine-self perspective blocks ("Imagine that these situations are happening to you"), and for the imaging-other perspective blocks ("Imagine that these situations are happening to someone else"). A colored border (blue or yellow) around the stimuli was used to further cue participants about which perspective to employ. Block order was pseudo-randomized across participants. Painful and non-painful scenarios were randomized within each block. Post-scan debriefings were conducted to make sure that subjects did follow the perspective-taking instructions.

\section{MRI ACQUISITION}

Scanning was conducted on a 1.5 Tesla Siemens Magnetom Avanto mobile unit equipped with advanced SQ gradients and a twelve element head coil. Functional images were collected using an EPI gradient-echo pulse sequence with TR/TE $=2000 / 39 \mathrm{~ms}$, flip angle $=90^{\circ}$, field of view $=240 \times 240 \mathrm{~mm}$, matrix $=64 \times$ $64 \mathrm{~cm}$, in-plane resolution $=3.4 \times 3.4 \mathrm{~mm}$, slice thickness $=$ $5 \mathrm{~mm}$, and 30 slices, full-brain coverage. Task presentation was implemented using the commercial software package E-Prime (Psychology Software Tools, Inc., Pittsburgh PA).

High-resolution T1-weighted structural MRI scans were acquired using a multiecho MPRAGE pulse sequence (repetition time $=2530 \mathrm{~ms}$, echo times $=1.64 \mathrm{~ms}, 3.50 \mathrm{~ms}, 5.36 \mathrm{~ms}$, $7.22 \mathrm{~ms}$, inversion time $=1100 \mathrm{~ms}$, flip angle $=7^{\circ}$, slice thickness $=1.3 \mathrm{~mm}$, matrix size $=256 \times 256$ ) yielding 128 sagittal slices with an in-plane resolution of $1.0 \times 1.0 \mathrm{~mm}$.

\section{IMAGE PROCESSING AND ANALYSIS}

Functional images were processed with SPM8 (Wellcome Department of Imaging Neuroscience, London, UK) in Matlab (Mathworks Inc., Sherborn, MA, USA). For each participant, functional data were realigned to the first image acquisition of the series and re-sampled to a voxel size of $2 \times 2 \times 2 \mathrm{~mm} 3$. Structural T1 images were co-registered to the mean functional image and segmented using the "New Segment" routine. A group-level structural template and individual flow fields were created using DARTEL, and the flow fields were in turn were used to spatially normalize functional images to standard MNI space. Data were smoothed with an $8 \mathrm{~mm}$ fullwidth at half maximum (FWHM) isotropic Gaussian kernel. Three participants were eliminated from further analysis due to issues related to movement or image quality, leaving $N=121$ ( $n=40,47,37$ for low, intermediate, and high psychopathy, respectively).

Statistics were calculated at the first level using the general linear model. The design matrix included three regressors for each stimulus category (detailed above), representing the event onsets and their time and dispersion derivatives. Movement parameters from the realignment output were included as regressors of no interest. All participants were entered into a second-level pooled analysis, and full brain activations were thresholded voxelwise at $p<0.001$ and with an extent threshold based on Gaussian 
random fields set to control the whole-brain family-wise error rate $(\mathrm{FWE})$ at $p<0.05$.

Second-level analyses were conducted by comparing the extremes of the sample distribution of PCL-R scores, and then as a continuous regressor using the entire sample. Participants with PCL-R total score at or above 30 were selected for the psychopathy group, while participants scoring at 20 or below comprised the incarcerated control group. For these analyses, regions of interest (ROIs) were defined using the MarsBar ROI toolbox. We focused on brain regions that were of maximal importance to the hypotheses under investigation, informed by the existing literature on empathy for pain in particular from a meta-analysis of 32 fMRI studies of empathy for pain (Lamm et al., 2011). MNI coordinates were selected from a previous fMRI study of empathy for pain in 80 male incarcerated participants (Decety et al., 2013). That study employed the same 1.5 mobile MRI scanner, and exposed the participants (from a different North American prison) to visual stimuli depicting bodily physical pain and videos of facial expressions of pain. ROI data are reported for significant contrast image peaks within $10 \mathrm{~mm}$ of these a priori coordinates (FWE-corrected $p<0.05$ ). Beyond existing literature on the processing of empathy-inducing stimuli in healthy populations, there may be additional cortical or subcortical brain regions that contribute to abnormal processing of these regions in psychopathy. For instance, the ventral striatum has been found to be overreactive in adolescents with conduct disorder as well as sexual sadists (Decety et al., 2009; Harenski et al., 2012). Therefore, coordinates for the ventral striatum were selected from a recent meta-analysis of fMRI studies (Diekhof et al., 2012).

To explore the extent to which results found in the groupwise analysis are driven by PCL-R Factor 1, Factor 2, or both, the regions reported above were tested for significant correlation with PCL-R factor scores. Corresponding $t$-values for sub-factor covariates within $10 \mathrm{~mm}$ of the ROIs above, if significant, were reported for each factor and task.

\section{FUNCTIONAL CONNECTIVITY}

Effective connectivity using psychophysiological interaction (PPI, Gitelman et al., 2003) was used to examine the effective connectivity from the anterior insula during imagine-first and imagine-third perspective taking conditions. The right anterior insula was selected because of its role in affective processing and attention. This polysensory region is considered as the integral hub of a salience network, which assists target brain regions in the generation of appropriate behavioral responses to salient stimuli (Menon and Uddin, 2010). Under the hypothesis that high psychopathy may result from a systemic brain deficit which is reflected in abnormal functional-connectivity patterns while imagining pain, we compared effective connectivity in imagine-self perspective and imagine-other perspective conditions between low- and high-psychopathy groups. Because of the importance of the amygdala reactivity (or the lack thereof) in psychopathy, we also ran a similar PPI analysis seeded in the right amygdala.

The time series of the first eigenvariates of the BOLD signal were temporally filtered, mean corrected, and deconvolved to generate the time series of the neuronal signal for the source region - the insula - as the physiological variable in the PPI. The psychological variable represented the time course of the contrast between painful and non-painful trials. An additional regressor represented the interaction of the psychological and physiological factors. These regressors were convolved with the canonical HRF and entered into the regression model. The interaction term in the resulting SPM showed areas with selective connectivity to the insula across the psychological contrast of pain vs. no pain. The PPI analysis was performed for each subject, and the resulting images of contrast estimates were entered into a random-effects group analysis. Second-level analysis results are reported at a voxelwise statistical cutoff of $p<0.001$ and a spatial extent threshold of $k>10$ voxels.

\section{RESULTS}

The entire sample of 121 participants (regardless of their psychopathy level) showed significant neuro-hemodynamic increase in the network of regions involved in the actual experience of physical pain under the imagine-self trials $(k>10, p<$ 0.05 , FWE corrected). This network includes the anterior insula (aINS), anterior midcingulate cortex (aMCC), supplementary motor area (SMA), inferior frontal gyrus (IFG), dorsomedial prefrontal cortex (dmPFC), mPFC, and somatosensory cortex, in both hemispheres (Table 1). In addition, signal change was detected in the left striatum and right amygdala.

When participants adopted the imagine-other perspective, a similar network was implicated, except for the right amygdala (Table 2). The only additional regions activated were the pSTS and $\mathrm{mPFC}$ in the right hemisphere. When imagine-other perspective was contrasted with imagine-self perspective, bilateral

Table 1 | Imagine-self perspective.

\begin{tabular}{llrrrl}
\hline \multirow{2}{*}{ Region of interest } & \multicolumn{3}{c}{ MNI coordinates } & \multirow{2}{*}{ Peak $\boldsymbol{T}$} \\
\cline { 3 - 4 } & & $\boldsymbol{x}$ & $\boldsymbol{y}$ & $\boldsymbol{z}$ & \\
\hline $\mathrm{L}$ & Anterior insula & -34 & 20 & 2 & 6.59 \\
$\mathrm{R}$ & Anterior insula & 44 & 14 & 0 & 5.28 \\
$\mathrm{~L}$ & Supramarginal gyrus & -58 & -28 & 32 & 6.81 \\
$\mathrm{R}$ & Supramarginal gyrus & 58 & -24 & 34 & 6.86 \\
$\mathrm{~L}$ & Supplementary motor area & -4 & 12 & 60 & 6.38 \\
$\mathrm{R}$ & Supplementary motor area & 6 & 10 & 60 & 6.35 \\
$\mathrm{~L}$ & Anterior midcingulate cortex & -6 & 20 & 38 & 6.12 \\
$\mathrm{R}$ & Anterior midcingulate cortex & 4 & 18 & 40 & 5.29 \\
$\mathrm{~L}$ & Dorsomedial prefrontal cortex & -8 & 54 & 14 & 5.87 \\
$\mathrm{R}$ & Dorsomedial prefrontal cortex & 4 & 56 & 18 & 5.29 \\
$\mathrm{R}$ & Lateral orbitofrontal cortex & 44 & 30 & -4 & 5.69 \\
$\mathrm{~L}$ & Inferior frontal gyrus & -38 & 28 & 4 & 6.10 \\
$\mathrm{R}$ & Inferior frontal gyrus & 54 & 12 & 8 & 5.52 \\
$\mathrm{~L}$ & Inferior parietal lobule & -44 & -54 & 38 & 4.73 \\
$\mathrm{R}$ & Inferior temporal gyrus & 46 & -66 & -12 & 5.43 \\
$\mathrm{R}$ & Amygdala & 20 & -4 & -14 & $3.72 *$
\end{tabular}

Pooled group results for all participants $(n=121)$.

All clusters are significant at FWE-corrected $p<0.05$ (cutoff, $t=4.72$ ), except those marked with a star, which are significant at uncorrected $p<0.0001$. L, left hemisphere; $R$, right hemisphere. 
Table 2 | Imagine-other perspective.

\begin{tabular}{llcccc}
\hline \multirow{2}{*}{ Region of interest } & \multicolumn{3}{c}{ MNI coordinates } & \multirow{2}{*}{ Peak T } \\
\cline { 3 - 5 } & & $\boldsymbol{x}$ & $\boldsymbol{y}$ & $\boldsymbol{z}$ & \\
\hline $\mathrm{L}$ & Anterior insula & -46 & 6 & -6 & 7.66 \\
$\mathrm{R}$ & Anterior insula & 34 & 28 & 8 & 5.45 \\
$\mathrm{~L}$ & Supramarginal gyrus & -56 & -36 & 36 & 7.21 \\
$\mathrm{R}$ & Supramarginal gyrus & 58 & -28 & 28 & 5.62 \\
$\mathrm{~L}$ & Supplementary motor area & -4 & 12 & 58 & 5.34 \\
$\mathrm{R}$ & Supplementary motor area & 6 & 10 & 58 & 6.41 \\
$\mathrm{~L}$ & Anterior cingulate cortex & -4 & 24 & 26 & 5.67 \\
$\mathrm{~L}$ & Anterior midcingulate cortex & -6 & 14 & 38 & 6.43 \\
$\mathrm{R}$ & Anterior midcingulate cortex & 0 & -10 & 34 & $3.93^{*}$ \\
$\mathrm{~L}$ & Dorsolateral prefrontal cortex & -42 & 40 & 10 & 7.07 \\
$\mathrm{R}$ & Dorsolateral prefrontal cortex & 48 & 30 & 0 & 5.52 \\
$\mathrm{~L}$ & Dorsomedial prefrontal cortex & -8 & 56 & 26 & 4.72 \\
$\mathrm{R}$ & Ventromedial prefrontal cortex & 8 & 54 & 2 & $4.04^{*}$ \\
$\mathrm{~L}$ & Inferior frontal gyrus & -52 & 8 & 6 & 10.16 \\
$\mathrm{R}$ & Inferior frontal gyrus & 50 & 12 & 4 & 7.24 \\
$\mathrm{~L}$ & Post. Superior temporal sulcus & -48 & -44 & 10 & $3.54^{*}$ \\
$\mathrm{R}$ & Post. Superior temporal sulcus & 50 & -36 & 2 & 5.03 \\
$\mathrm{~L}$ & Inferior parietal lobule & -44 & -34 & 34 & 5.17 \\
$\mathrm{~L}$ & Dorsal striatum & -12 & 0 & 4 & 5.79 \\
\hline
\end{tabular}

Pooled group results for all participants $(n=121)$. All clusters are significant at FWE-corrected $p<0.05$ (cutoff, $t=4.72$ ), except those marked with a star, which are significant at uncorrected $p<0.0001$.

activation was detected in the superior parietal cortex $(-23,-52$, 60 and $27,-44,59)$, superior frontal gyrus $(-21,-7,52$ and $26,-8,52)$, and dorsal striatum $(-6,4,12$ and $9,4,11)$. No significant signal increase was detected for the reverse contrast.

\section{REGION OF INTEREST ANALYSES}

Results from the ROI analyses are presented in Table 3. When participants with low scores on the PCL-R were compared with individuals scoring high on the PCL-R, the $\operatorname{mPFC}(-12,52$, 8) was activated during imagine-self perspective. A cluster of significant hemodynamic increase was found in the OFC. The opposite contrast (high psychopathy $>$ low psychopathy) showed increased signal in the aMCC, SMA, right aINS, IFG, and right pSTS/TPJ. All participants showed significant response in the right amygdala during imagine-self perspective (Figure 1).

During the imagine-other perspective, individuals with low scores on the PCL-R compared with individuals with high scores on the PCL-R, showed greater signal change in the SMA, right $\mathrm{mPFC}$, intraparietal sulcus, precentral gyrus, and parahippocampal gyrus/amygdala, pSTS, dorsal aINS and dorsal ACC. In participants with high scores on the PCL-R, the imagine-other perspective was associated with greater activation in the dlPFC and ventral striatum $(p<0.001)$, when compared to low-scoring incarcerated controls.

\section{CORRELATIONS BETWEEN PCL-R SCORES AND ROIS}

The hemodynamic response in the aINS was significantly greater in individuals scoring high on psychopathy (total PCL-R score) during imagine-self perspective, and the reverse was found for imagine-other perspective (Figure 2 ). Factor 2 positively correlated with the activity in aINS during imagine-self perspective $(r=0.372, p=0.016)$, whereas it negatively correlated with aINS activity during imagine-other perspective $(r=-0.254, p=$ $0.01)$. Factor 1 was negatively correlated with response in aINS during third-person perspective $(r=-0.272, p=0.01)$. Activity in the dmPFC was negatively associated with both Factor 1 $(r=-0.24, p<0.01)$ and Factor $2(r=-0.237, p=0.01)$ during imagine-self perspective. The hemodynamic response in the dlPFC was positively correlated with both Factor $1(r=0.288$, $p<0.01)$ and Factor $2(r=0.274, p<0.01)$ during imagineother perspective. The response in the ventral striatum during imagine-other perspective significantly correlated with scores on Factor $1(r=0.212, p<0.02$, see Figure 3). Finally, response in the right amygdala $(26,2,-18)$ showed a negative correlation with Factor $1(r=-0.258, p=0.04)$ during imagine-other perspective. No significant correlation was found in imagine-self perspective with either Factors 1 and 2. See Table 3 for a complete list of results.

\section{EFFECTIVE CONNECTIVITY ANALYSES}

Functional connectivity analyses seeded in the anterior insula revealed distinct patterns in functional coupling between the low- and high-psychopathy groups. During imagine-self perspective, individuals scoring low on the PCL-R showed a negative connectivity between the aINS and the hippocampus and the OFC (Figure 4). In the high psychopathy group, there was only significant functional connectivity between the aINS and the right pSTS. During imagine-other perspective, low-psychopathy participants had significant effective connectivity between the aINS and posterior cingulate cortex and dIPFC (Figure 5). In high-scoring participants, negative connectivity was found between aINS and the right OFC and posterior cingulate cortex.

Functional connectivity analyses seeded in the right amygdala showed distinct patterns of co-variations depending on the perspective adopted in controls vs. psychopaths. During imagineself perspective, controls exhibited a significant negative coupling between the amygdala and ventral and mPFC, while participants with high scores on the PCL-R showed a positive coupling with the pSTS/TPJ, ventral and mPFC, and dlPFC (Figure 6). During imagine-other perspective, the reverse pattern of functional connectivity was observed. Low psychopathy was associated with greater positive coupling with the OFC, whereas the high psychopathy showed a negative coupling with the OFC and dIPFC (Figure 7).

\section{DISCUSSION}

Perspective taking while observing or imagining other's feelings has been described as an empathic attentional set that facilitates other-oriented emotional and motivational responses congruent with the perceived welfare of that person (Van Lange, 2008; Batson, 2012). To examine the extent to which affective reactions can be evoked or modulated by perspective taking in individuals with psychopathy, incarcerated participants with different levels on the PCL-R were scanned while viewing stimuli depicting 
Table 3 | Groupwise results and factor sub-score covariates for imagine-self and imagine-other perspectives.

\begin{tabular}{|c|c|c|c|c|c|c|c|c|c|c|c|c|}
\hline Region of interest & \multicolumn{3}{|c|}{ MNI coordinates } & Peak $T$ & \multicolumn{3}{|c|}{ Factor 1} & Peak $T$ & \multicolumn{3}{|c|}{ Factor 2} & Peak $T$ \\
\hline
\end{tabular}

\section{IMAGINE-SELF PERSPECTIVE}

Controls > Psychopaths

\begin{tabular}{|c|c|c|c|c|c|c|c|c|c|c|c|c|c|}
\hline $\mathrm{R}$ & Orbitofrontal cortex & 14 & 58 & -2 & 3.28 & & & & n.s. & 24 & 54 & 2 & -2.15 \\
\hline$L$ & dIPFC & -12 & 52 & 8 & 3.40 & -16 & 54 & 6 & -3.12 & -14 & 52 & 4 & -2.44 \\
\hline $\mathrm{L}$ & Periaqueductal gray & 0 & -28 & -14 & 3.23 & & & & n.s. & -4 & -24 & -14 & -3.01 \\
\hline
\end{tabular}

Psychopaths $>$ Controls

\begin{tabular}{|c|c|c|c|c|c|c|c|c|c|c|c|c|c|}
\hline $\mathrm{R}$ & Inferior frontal gyrus & 50 & 26 & 8 & 2.65 & 48 & 26 & 10 & 2.99 & 52 & 24 & 8 & 2.83 \\
\hline$L$ & Anterior midcingulate cortex & -4 & 8 & 34 & 2.82 & -2 & 8 & 30 & 3.31 & & & & n.s. \\
\hline $\mathrm{R}$ & Anterior midcingulate cortex & 4 & 10 & 32 & 3.01 & 4 & 10 & 30 & 2.93 & 6 & 6 & 32 & 3.38 \\
\hline$L$ & Supplementary motor area & -10 & 2 & 50 & 2.49 & -6 & 2 & 54 & 2.37 & -8 & 6 & 44 & 3.18 \\
\hline $\mathrm{R}$ & Anterior insula & 38 & 20 & 12 & 2.74 & 32 & 14 & 4 & 2.03 & 34 & 20 & 8 & 2.73 \\
\hline $\mathrm{R}$ & pSTS & 44 & -48 & 14 & 2.41 & 46 & -48 & 16 & 2.61 & 44 & -50 & 18 & 3.44 \\
\hline
\end{tabular}

Controls > Psychopaths

\begin{tabular}{|c|c|c|c|c|c|c|c|c|c|c|c|c|c|}
\hline$R$ & Inferior frontal gyrus & 44 & 26 & 2 & 2.25 & 40 & 30 & 2 & -2.62 & 38 & 28 & 10 & -2.59 \\
\hline $\mathrm{R}$ & Anterior midcingulate cortex & 6 & 18 & 34 & 2.21 & & & & n.s. & 8 & 16 & 34 & -2.44 \\
\hline $\mathrm{R}$ & mPFC & 16 & 32 & 12 & 3.58 & 16 & 32 & 12 & -3.88 & 12 & 40 & 14 & -2.67 \\
\hline $\mathrm{L}$ & Anterior insula & -44 & 14 & 4 & 2.25 & -44 & 14 & 4 & -2.48 & -44 & 12 & 0 & -2.21 \\
\hline $\mathrm{R}$ & Anterior insula & 34 & 30 & 4 & 3.07 & 42 & 14 & 2 & -2.54 & 42 & 10 & 2 & -2.25 \\
\hline$L$ & Supplementary motor area & -6 & 16 & 54 & 3.04 & -8 & 20 & 54 & -2.3 & -8 & 20 & 54 & -4.14 \\
\hline $\mathrm{R}$ & Supplementary motor area & 8 & 24 & 46 & 2.69 & 6 & 26 & 46 & 2.13 & 4 & 18 & 52 & -2.22 \\
\hline $\mathrm{R}$ & pSTS & 50 & -52 & 22 & 3.09 & 50 & -52 & 20 & -3.04 & 52 & -50 & 16 & -2.23 \\
\hline $\mathrm{R}$ & Inferior parietal lobule & 44 & -32 & 22 & 2.99 & 42 & -32 & 22 & -3.14 & 48 & -32 & 26 & -3.13 \\
\hline $\mathrm{L}$ & Inferior parietal lobule & -48 & -36 & 22 & 2.81 & -44 & -38 & 22 & -3.19 & -46 & -38 & 22 & -3.24 \\
\hline $\mathrm{R}$ & Putamen & 30 & 8 & 2 & 3.5 & 30 & 8 & 0 & -2.7 & 30 & 8 & 2 & -2.77 \\
\hline $\mathrm{L}$ & Putamen & -14 & 10 & -2 & 2.48 & -12 & 8 & 0 & -2.56 & & & & n.s. \\
\hline
\end{tabular}

Psychopaths $>$ Controls

\begin{tabular}{|c|c|c|c|c|c|c|c|c|c|c|c|c|c|}
\hline $\mathrm{R}$ & dIPFC & 28 & 48 & 14 & 3.28 & 30 & 48 & 12 & 2.33 & 26 & 50 & 14 & 3.60 \\
\hline $\mathrm{L}$ & Inferior temporal gyrus & -50 & -38 & -18 & 2.62 & -52 & -40 & -16 & 3.52 & -52 & -40 & -14 & 3.55 \\
\hline $\mathrm{R}$ & Ventral striatum & 10 & 16 & -6 & 3.55 & 10 & 16 & -6 & 3.18 & 12 & 16 & -4 & 3.84 \\
\hline
\end{tabular}

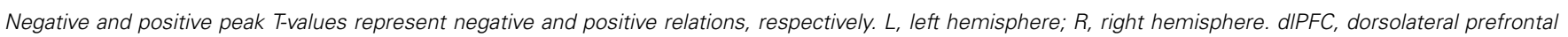
cortex; MPFC, medial prefrontal cortex; pSTS, posterior superior temporal sulcus.

bodily injuries and instructed to imagine these situations as either happening to themselves or to someone else.

At the group level, collapsed across the PCL-R scores $(n=$ 121 ), both conditions of imagine-self and imagine-other in pain were associated with signal increase in brain regions implicated in the perception of pain and distress, when viewing body parts suffering injuries or facial expressions of pain (Jackson et al., 2006; Lamm et al., 2007, 2011; Decety and Porges, 2011; Bruneau et al., 2012). In healthy participants, activity in this network, which includes the aINS, thalamus, aMCC, IFG, and somatosensory cortex, has been interpreted as a form of somatosensory resonance, or shared neural representations with the pain of others, providing an implicit intersubjective affective knowledge (Decety and Jackson, 2004; Singer and Decety, 2011; Zaki and Ochsner, 2012). However, these vicariously instigated activations of the so-called "pain matrix" are not specific to the sensory qualities of pain, but instead are associated with more general survival mechanisms such as aversion and withdrawal when exposed to danger and threat (Benuzzi et al., 2008; Decety, 2010). In fact, based on a systematic review of electroencephalographic and functional MRI studies that examined neural response triggered by nociceptive stimuli, activity of this cortical network seems to reflect a system involved in detecting, processing, and reacting to the occurrence of salient sensory events regardless of the sensory channel through which these events are conveyed (Legrain et al., 2011).

Interestingly and quite surprisingly, the hemodynamic response in aINS and aMCC, regions considered as pivotal in the affective component of empathy, was highest in high psychopaths during imagine-self perspective, replicating the results of a recent study of pain empathy in criminal psychopaths that reported 


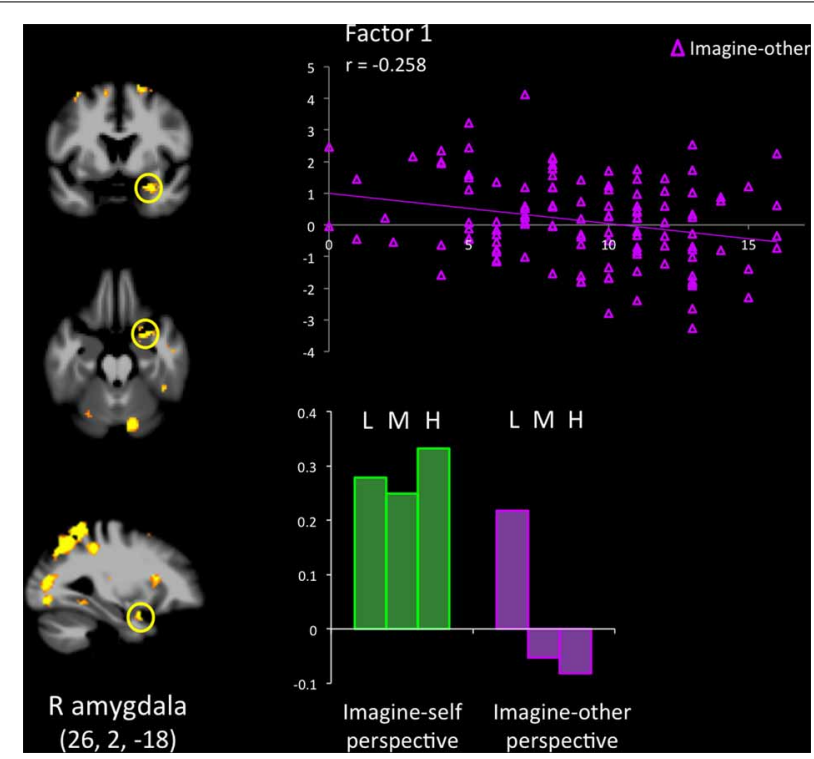

FIGURE 1 | Response in the right amygdala across groups of low (L), medium (M), and high (H) psychopathy (on total PCL-R scores) participants, when they adopted an imagine-self and an imagine-other affective perspective while viewing bodily injuries. Groupwise effects (bars at the bottom of the figure) are expanded to show the contribution of continuous PCL-R subscores on Factor 1, which encompasses the emotional/interpersonal features of psychopathy.

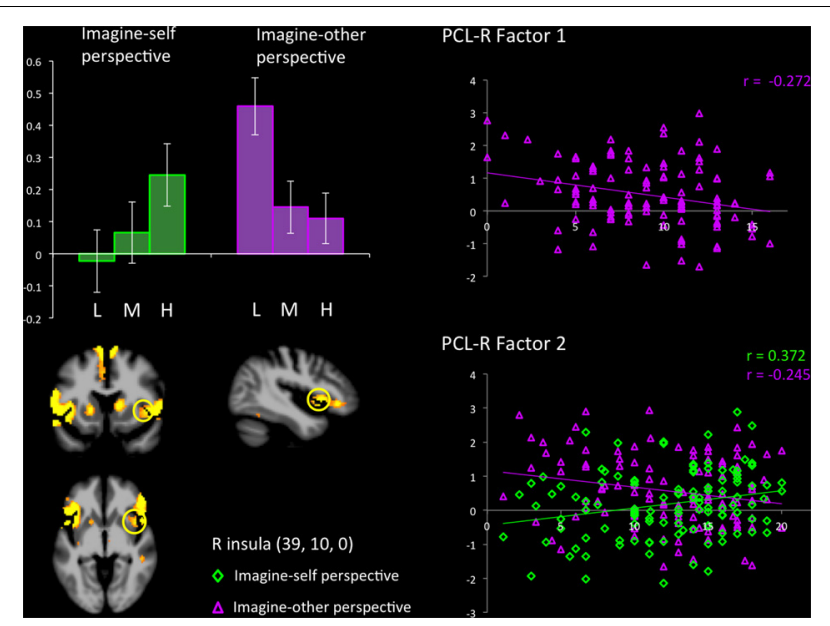

FIGURE 2 | Response in the right anterior insula across groups (L, low; M, medium; $\mathrm{H}$, high on total PCL-R scores) during imagine-self and imagine-other perspectives in participants viewing bodily injuries. Groupwise effects seen in (bar graph) are expanded to show the contribution of Factors 1 and 2 from PCL-R subscores.

greater activation in the insula, which was positively correlated with scores on both PCL-R factors 1 and 2 (Decety et al., 2013) (Figure 2). The aINS and aMCC are the two regions that have been most reliably activated in fMRI studies of pain empathy with healthy subjects (Valentini, 2010; Lamm et al., 2011). This finding does not support the view that psychopaths do not resonate when exposed aversive stimuli such as pain, or at least they are

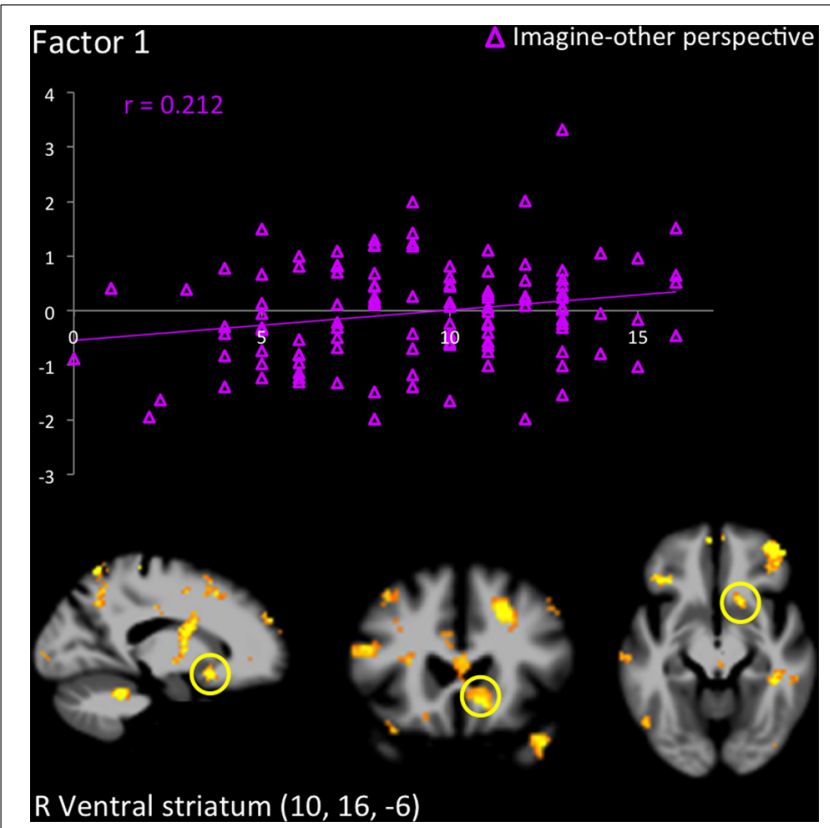

FIGURE 3 | Response in the right ventral striatum in participants scoring high on the PCL-R $(\geq 30)$ when they imagined another person in pain, and correlation with scores on Factor 1.

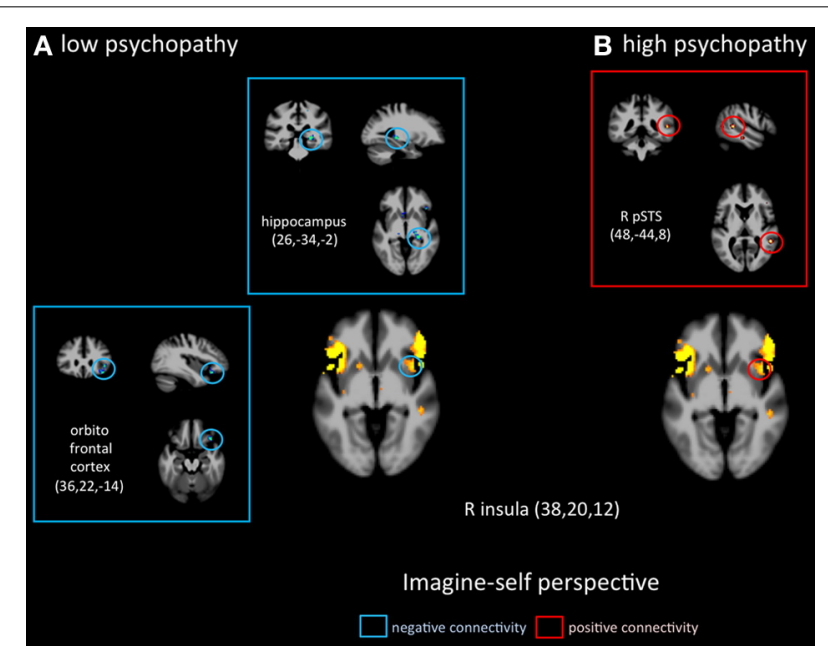

FIGURE 4 | Functional connectivity analyses, seeded in the anterior insula in participants with the lowest scores on the PCL-R $(\leq 20)$ and participants with the highest scores on the PCL-R $(\geq 30)$ during imagine-self perspective.

not totally blunted when they take a first-person perspective. This finding also raises an interesting question: whether or not sensorimotor resonance (underpinned by the mirror neuron system involved in perception-action coupling) is the mechanism that facilitates emotion contagion and empathic arousal. Psychopaths are characterized by a lack of affective empathy, but there is little evidence that they show a deficit in sensorimotor resonance (Blair, 2011; Decety, 2011b). For instance, a transcranial magnetic stimulation study demonstrated increased 


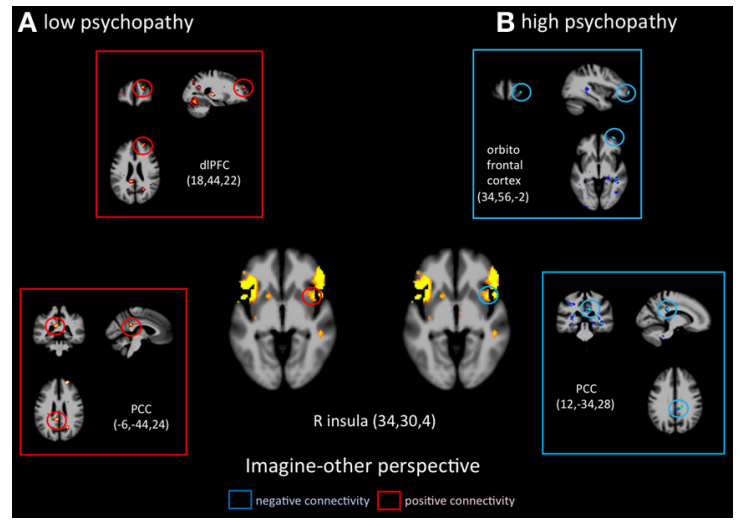

FIGURE 5 | Functional connectivity analyses, seeded in the anterior insula in participants with the lowest scores on the PCL-R and participants with the highest scores on the PCL-R $(>30)$ during imagine-other perspective.

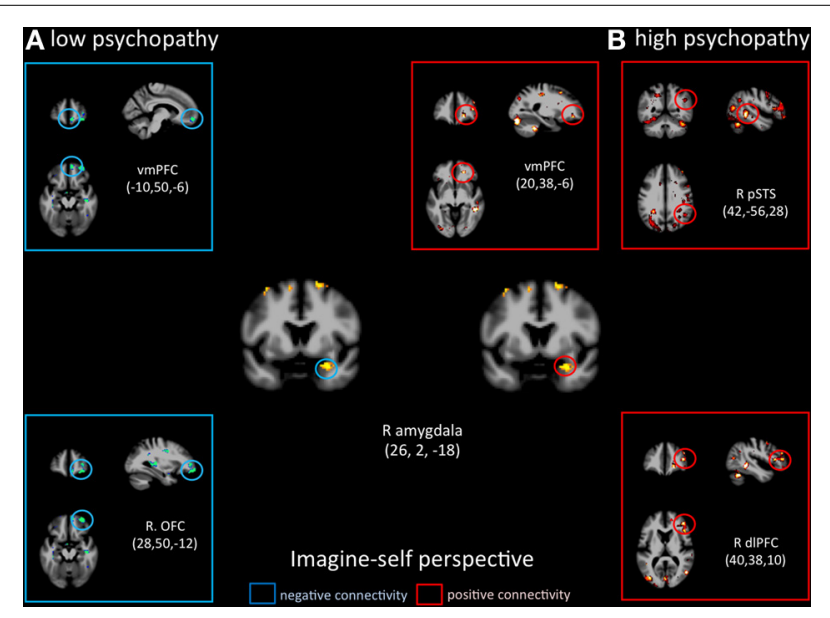

FIGURE 6 | Functional connectivity analyses, seeded in the right amygdala in participants with the lowest scores on the PCL-R $(\leq 20)$ and participants with the highest scores on the PCL-R $(\geq 30)$ during imagine-self perspective.

sensorimotor resonance to painful hand-pricking videos in college students scoring high on the psychopathic personality inventory (PPI), as compared to students who score low on the PPI (Fecteau et al., 2008). Juvenile incarcerated psychopaths showed greater sensorimotor resonance as measured by EEG and suppression of the $m u$ rhythm when they viewed visual stimuli depicting people being physically injured, despite a lack of affective arousal to the same stimuli as measured by the N120 ERP component (Cheng et al., 2012). Children with aggressive conduct disorder and psychopathic tendencies and incarcerated psychopaths exhibit typical (Marsh et al., 2013) or even stronger activation in the somatosensory cortex than control participants when they watched scenarios depicting people in pain (Decety et al., 2009, 2013), all of which does not suggest an impairment in somatosensory responses to others' pain. Our finding that participants scoring high on psychopathy activate the pain

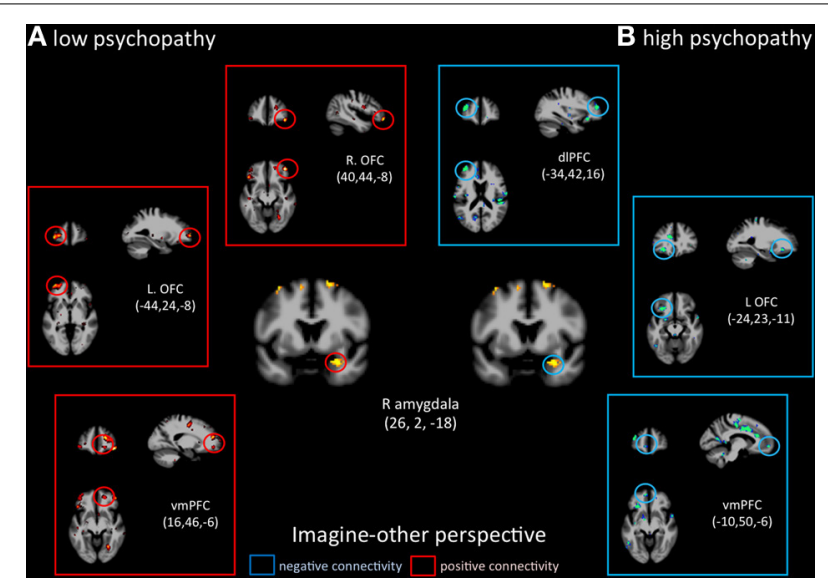

FIGURE 7 | Functional connectivity analyses, seeded in the right amygdala in participants with the lowest scores on the PCL-R and participants with the highest scores on the PCL-R $(>30)$ during imagine-other perspective.

network during imagine-self perspective fits well with studies showing that individuals with psychopathy may up-regulate emotional (at least for fear) processing when attention to salient stimuli is particularly engaged (Newman and Lorenz, 2003), and this may be the case for pain.

Furthermore, and as expected, the lower the participants scored on Factors 1 and 2 of the PCL-R, the higher the activity in the aINS during imaging-other perspective. This indicates that more vicarious experience was elicited in control participants when they imagined another in pain, and the opposite pattern (low activation in the aINS) was found in participants who scored high on psychopathy. In addition, functional connectivity analyses, seeded in the right aINS during imagine-self perspective negatively co-varied with activation in the hippocampal gyrus and OFC in control participants (low on psychopathy), and was positively coupled with the right pSTS region in psychopaths. During imagine-other perspective, the aINS positively covaried with activity in the right dlPFC and PCC in controls, and negatively with the OFC and PCC in high psychopaths. Altogether, the hemodynamic response in the aINS shows distinct profiles of activation depending on whether participants adopted an imagine-self or imagine-other perspective taking. These results from the imagine-other perspective condition support two recent functional neuroimaging studies in children with conduct disorder (Lockwood et al., 2013; Marsh et al., 2013). Both studies reported a reduced response in the aINS and ACC when the children viewed pictures of others in pain. Furthermore, a negative association between callous traits and the aINS/ACC was found. The fact that individuals with high scores on the PCL-R showed a reduced response when imagining the pain of another suggests a specific deficit in affective processing in a region considered as a critical hub to integrate salient stimuli and events with visceral and autonomic information (Menon and Uddin, 2010).

Signal change in the right amygdala was detected during imagine-self perspective in all participants, and during imagineother perspective in controls. The hemodynamic response in 
the amygdala was inversely correlated with individual scores on PCL-R Factor 1 during imagine-other perspective. This is in line with most neuroimaging studies of psychopathy that documented reduced amygdala response to fearful and aversive stimuli (Marsh and Blair, 2008; Harenski et al., 2009). This finding is consistent with the notion that psychopaths lack the ability to be responsive to, or aroused by distress cues, and therefore are not sensitive to signs of vulnerability. A recent fMRI study in youths with psychopathic traits also reported reduction in the amygdala and insula when they imagined physical injuries to others, but not their own pain (Marsh et al., 2013).

It is very interesting to note that imagine-self perspective was associated with activity in the amygdala in psychopaths when they focus on their own affective reaction. While most studies report a reduced response in the amygdala in psychopaths, an fMRI study conducted on a small number psychopaths and controls found increased activation in the right amygdala in the psychopath group with respect to controls when viewing negative IAPS pictures (Müller et al., 2003), indicating that the role of the amygdala in psychopathy may not be straightforward, nor its lateralization. A meta-analysis of 67 neuroimaging studies reported that the lateralization of activation in the amygdala was explained by differences in temporal dynamics and/or habituation rates, namely a short-duration response in the right amygdala and a more sustained one in the left (Sergerie et al., 2008). It is however difficult to interpret the amygdala activation during imagine-self perspective further without a more fine-grain analysis of amygdala sub-nuclei and their anatomical connectivity, which helps determine their function (Saygin et al., 2011). With this caveat in mind, it is important to note that functional connectivity analyses, seeded in the right amygdala, demonstrated very different patterns of connectivity depending on the perspective taking strategy (imagine-self vs. imagineother) and participants (low vs. high psychopaths). The response in the right amygdala was negatively coupled with activity in the OFC in controls and positively correlated with the OFC and dIPFC and pSTS in high psychopathy during imagine-self perspective (Figure 3). The exact reverse functional connectivity was detected during imagine-other perspective (Figure 4). This finding specifically points to amygdala-OFC interactions as being an important neural mechanism that underlies the outcome of perspective taking in psychopathy. It seems to indicate that during imagine-self perspective, individuals with psychopathy elicit amygdala-OFC coupling but fail to do so during imagine-other perspective. Such a failure to recruit the OFC during third-person perspective taking supports the dysfunction of this neural pathway in response to distress cues of others in psychopaths. It has been argued that the integrated functioning of this circuit enables the basics of care-based morality, and that dysfunction within these regions in psychopathy means that reinforcementbased decision making, including moral decision making, and care base morality is impaired (Blair, 2007; Shamay-Tsoory et al., 2010; Marsh et al., 2011). One theory of the origin of empathic deficits in psychopathy is the failure during development to form stimulus-reinforcement associations connecting harmful or aggressive actions with the pain and distress of others (Kiehl,
2006; Glenn and Raine, 2009). It is worth mentioning that psychopathic traits are not exclusively associated with amygdala hyporeactivity. A study that included 200 young adults with self-reported psychopathy assessment found that amygdala reactivity to fearful facial expressions is negatively associated with the interpersonal facet of psychopathy, whereas reactivity to angry expressions is positively associated with the lifestyle facet (Carré et al., 2013).

Finally, the increase of activity in the ventral striatum during imagine-other perspective in psychopaths, which was predicted by their scores on Factor 1 of the PCL-R, is an intriguing finding. This could suggest that psychopaths not only experience blunted vicariously arousal to others' pain and reduced feelings of concern when adopting their perspective, but they may in fact find the distress of others pleasurable or positively arousing. The ventral striatum is selectively recruited during reward anticipation in healthy participants (Diekhof et al., 2012 for a meta-analysis). In adolescents with conduct disorder and psychopathic tendencies, an fMRI study found activation of the ventral striatum during the perception of pain in others (Decety et al., 2009). In healthy subjects, the ventral striatum has been associated with experiencing pleasure at others' misfortune (e.g., Dvash et al., 2010; Cikara et al., 2011). It has been suggested that neurons in the ventral striatum have access to central representations of reward and thereby participate in the processing of information underlying the motivational control of goal-directed behavior (Schultz et al., 1992). Activation of the ventral striatum while imaging another in physical pain was correlated with PCL-R Factor 1, and not Factor 2. Abnormalities in the ventral and dorsal striatum are considered to play a key role in the etiology of psychopathic traits (Buckholtz et al., 2010; Carré et al., 2013).

\section{CONCLUSION}

There is general consensus among theorists that the ability to adopt and entertain the psychological perspective of others has a number of important consequences, including empathic concern (e.g., Blair, 2007; Batson, 2009; Decety and Svetlova, 2012). Adopting the perspective of another is a powerful way to place oneself in the situation or emotional state of that person (Batson, 2011). Our results demonstrate that while individuals with psychopathy exhibited a strong response in pain-affective brain regions when taking an imagine-self perspective, they failed to recruit the neural circuits that are were activated in controls during an imagine-other perspective, and that may contribute to lack of empathic concern. Finally, this atypical pattern of activation and effective connectivity associated with perspective taking manipulations may inform intervention programs in a domain where therapeutic pessimism is more the rule than the exception (Salekin, 2002). Altered connectivity may constitute novel therapeutic targets for interventions. Both cognitive and pharmacotherapy interventions may restore connectivity patterns (Crocker et al., 2013). Imagining oneself in pain or in distress may trigger a stronger affective reaction than imagining what another person would feel, and this could be used with some psychopaths in cognitive-behavior therapies as a kick-starting technique 
for eliciting emotional tagging of different outcomes of interpersonal situations.

\section{ACKNOWLEDGMENTS}

This study was supported by NIMH R01 grant 1R01MH08752501A2 (J. Decety, PI) and by NIMH R01 grant MH070539-01

\section{REFERENCES}

Anderson, N. E., and Kiehl, K. A. (2011). The psychopath magnetized: insights from brain imaging. Trends Cogn. Sci. 16, 52-60.

Barrett-Lennard, G. T. (1981). The empathy cycle: refinement of a nuclear concept. J. Couns. Psychol. 28, 91-100. doi: 10.1037/0022-0167.28.2.91

Batson, C. D. (2009). "These things called empathy: eight related but distinct phenomena," in The Social Neuroscience of Empathy, eds J. Decety and W. Ickes (Cambridge: MIT Press), 3-15.

Batson, C. D. (2011). Altruism in Humans. New York, NY: Oxford University Press.

Batson, C. D. (2012). "The empathyaltruism hypothesis: issues and implications," in Empathy: From Bench to Bedside, ed J. Decety (Cambridge: MIT Press), 41-54.

Batson, C. D., Early, S., and Salvarini, G. (1997). Perspective taking: imagining how another feels versus imagining how you would feel. Pers. Soc. Psychol. Bull. 23, 751-758. 10.1177/0146167297237008.

Benuzzi, F., Lui, F., Duzzi, D., Nichelli, P. F., and Porro, C. A. (2008). Does it look painful or disgusting. Ask your parietal and cingulate cortex. J. Neurosci. 28, 923-931. doi: 10.1523/JNEUROSCI.4012-07.2008

Blair, R. J. R. (2005). Responding to the emotions of others: dissociating forms of empathy through the study of typical and psychiatric populations. Conscious. Cogn. 14, 698-718. doi: 10.1016/j.concog.2005.06.004

Blair, R. J. R. (2007). The amygdala and ventromedial prefrontal cortex in morality and psychopathy. Trends Cogn. Sci. 11, 387-392.

Blair, R. J. R. (2011). Should affective arousal be grounded in perception-action coupling. Emot. Rev. 3, 109-110. doi: 10.1177/1754073910384157

Blair, R. J. R., Jones, L., Clark, F., and Smith, M. (1997). The psychopathic individual: a lack of responsiveness to distress cues. Psychophysiology 34, 192-198. doi: 10.1111/j.14698986.1997.tb02131.x

Buckholtz, J. W., Treadway, M. T., Cowan, R. L., Woodward, N. D.,
Benning, S. D., Li, R., et al. (2010). Mesolimbic dopamine reward system hypersensitivity in individuals with psychopathic traits. Nat. Neurosci. 13, 419-421. doi: 10.1038/nn.2510

Brook, M., and Kosson, D. S. (2012). Impaired cognitive empathy in criminal psychopathy: evidence from a laboratory measure of empathic accuracy. J. Abnorm. Psychol. 122, 156-166. doi: 10.1037/a0030261

Bruneau, E. G., Pluta, A., and Saxe, R. (2012). Distinct roles of the shared pain and theory of mind networks in processing others' emotional suffering. Neuropsychologia 50, 219-231. doi: 10.1016/j.neuro

Carré, J. M., Hyde, L. W., Neumann, C. S., Viding, E., and Hariri, A. R. (2013). The neural signatures of distinct psychopathic traits. Soc. Neurosci. 8, 122-135. doi: 10.1080/17470919.2012.703623

Cheng, Y., Hung, A., and Decety, J. (2012). Dissociation between affective sharing and emotion understanding in juvenile psychopaths. Dev. Psychopathol. 24, 623-636. doi: 10.1017/S095457941200020X

Cikara, M., Botvinick, M. M., and Fiske, S. T. (2011). Us versus them: social identity shapes responses to intergroup competition and harm. Psychol. Sci. 22, 306-313. doi: 10.1177/0956797610397667

Craig, A. D. (2002). How do you feel. Interoception: the sense of the physiological condition of the body. Nat. Rev. Neurosci. 3, 655-666.

Crocker, L. D., Heller, W., Warren, S. L., O'Hare, A. J., Infantolino, Z. P., and Miller, G. A. (2013). Relationships among cognition, emotion, and motivation: implications for intervention and neuroplasticity in psychopathology. Front. Hum. Neurosci. 7:261. doi: 10.3389/fnhum.2013.00261

Cunningham, W. A., and Brosch, T. (2012). Motivational salience: amygdala tuning from traits, needs, values, and goals. Curr. Dir. Psychol. Sci. 21, 54-59. doi: $10.1177 / 0963721411430832$

Decety, J. (2010). To what extent is the experience of empathy psychologia.2011.11.008

and NIDA 1R01DA026505-01A1 (K. Kiehl, PI). Dr. J. Decety, Dr. C. Chen, Dr. C. Harenski, and Dr. K. Kiehl have no conflicts of interest to disclose. Dr. Decety takes full responsibility for the integrity of the data and the accuracy of the data analysis. All authors had full access to all the data in the study.

mediated by shared neural circuits. Emot. Rev. 2, 204-207. doi: $10.1177 / 1754073910361981$

Decety, J. (2011a). Dissecting the neural mechanisms mediating empathy. Emot. Rev. 3, 92-108. doi: $10.1177 / 1754073910374662$

Decety, J. (2011b). Promises and challenges of the neurobiological approach to empathy. Emot. Rev. 3, 115-116. doi $10.1177 / 1754073910384160$

Decety, J., and Hodges, S. D. (2006). "A social cognitive neuroscience model of human empathy," in Bridging Social Psychology: benefits of Transdisciplinary Approaches, ed P. A. M. van Lange (Mahwah, NJ: Lawrence Erlbaum Associates), 103-109.

Decety, J., and Jackson, P. L. (2004). The functional architecture of human empathy. Behav. Cogn. Neurosci. Rev. 3, 71-100. doi 10.1177/1534582304267187

Decety, J., Michalska, K. J., Akitsuki, Y., and Lahey, B. (2009). Atypical empathic responses in adolescents with aggressive conduct disorder: a functional MRI investigation. Biol. Psychol. 80, 203-211. doi 10.1016/j.biopsycho.2008.09.004

Decety, J., Michalska, K. J., and Kinzler K. D. (2012). The contribution of emotion and cognition to moral sensitivity: a neurodevelopmental study. Cereb. Cortex 22, 209-220. doi: 10.1093/cercor/bhr111

Decety, J., and Porges, E. C. (2011) Imagining being the agent of actions that carry different moral consequences: an fMRI study. Neuropsychologia 49, 2994-3001. doi: $\quad 10.1016 /$ j.neuropsychologia. 2011.06.024

Decety, J., Skelly, L. R., and Kiehl, K. A. (2013). Brain response to empathy-eliciting scenarios involving pain incarcerated individuals with psychopathy. JAMA Psychiatry 70, 638-645. doi: 10.1001/jamapsychiatry.2013.27

Decety, J., and Svetlova, M. (2012). Putting together phylogenetic and ontogenetic perspectives on empathy. Dev. Cogn. Neurosci. 2, 1-24. doi: 10.1016/j.den.2011.05.003

Diekhof, E. K., Kaps, L., Falkai, P., and Gruber, O. (2012). The role of the human ventral striatum and the medial orbitofrontal cortex in the representation of reward magnitude - an activation likelihood estimation meta-analysis of neuroimaging studies of passive reward expectancy and outcome processing. Neuropsychologia 50, 1252-1266. doi: $\quad 10.1016 / j$.neuropsychologia. 2012.02.007

Dolan, M., and Fullam, R. (2004). Theory of mind and mentalizing ability in antisocial personality disorder with and without psychopathy. Psychol. Med. 34, 1093-1102. doi: $10.1017 / S 0033291704002028$

Dvash, J., Gilam, G., Ben-Ze'ev, A., Hendler, T., and Shamay-Tsoory, S. G. (2010). The envious brain: the neural basis of social comparison. Hum. Brain Mapp. 31, 1741-1750.

Fecteau, S., Pascual-Leone, A., and Theoret, H. (2008). Psychopathy and the mirror neuron system: preliminary findings from a non-psychiatric sample. Psychiatry Res. 160, 137-144. doi: 10.1016/j.psychres.2007.08.022

Gitelman, D. R., Penny, W. D. Ashburner, J., and Friston, K. J. (2003). Modeling regional and psychophysiologic interactions in fMRI: the importance of hemodynamic deconvolution. Neuroimage 19, 200-207. doi: 10.1016/S1053-8119(03)00058-2

Gleichgerrcht, E., Torralva, T., Roca, M., Pose, M., and Manes, F. (2011). The role of social cognition in moral judgment in frontotemporal dementia. Soc. Neurosci. 6, 113-122. doi: 10.1080/17470919. 2010.506751

Glenn, A. L., and Raine, A. (2009). Psychopathy and instrumental aggression: evolutionary, neurobiological and legal perspectives. Int. J. Law Psychiatry 32, 253-258. doi: 10.1016/j.ijlp.2009.04.002

Halpern, J. (2012). "Clinical empathy in medical care," in Empathy: From Bench to Bedside, ed J. Decety (Cambridge: MIT Press), 229-244.

Hare, R. D. (2003). The Hare Psychopathy Checklist-Revised, Manual, 2nd Edn. Toronto, ON: Multi-Health Systems.

Harenski, C. L., Kim, S. H., and Hamann, S. (2009). Neuroticism 
and psychopathy predict brain activation during moral and nonmoral emotion regulation. Cogn. Affect. Behav. Neurosci. 9, 1-15. doi: 10.3758/CABN.9.1.1

Harenski, C. L., Thornton, D. M., Harenski, K. A., Decety, J., and Kiehl, K. A. (2012). Increased fronto-temporal activation during pain observation in sexual sadism. Arch. Gen. Psychiatry 69, 283-292. doi: 10.1001/archgenpsychiatry.2011.1566

Herpertz, S. C., and Sass, H. (2000). Emotional deficiency and psychopathy. Behav. Sci. Law 18, 567-580.

Hynes, C. A., Baird, A. A., and Grafton, S. T. (2006). Differential role of the orbitofrontal lobe in emotional versus cognitive perspective-taking. Neuropsychologia 44, 374-383. doi: 10.1016/j.neuropsychologia.2005. 06.011

Jackson, P. L., Brunet, E., Meltzoff, A. N., and Decety, J. (2006). Empathy examined through the neural mechanisms involved in imagining how I feel versus how you feel pain: an event-related fMRI study. Neuropsychologia 44, 752-761. doi: $\quad 10.1016 / j$.neuropsychologia. 2005.07.015

Kiehl, K. A. (2006). A cognitive neuroscience perspective on psychopathy: evidence for paralimbic system dysfunction. Psychiatry Res. 142, 107-128. doi: 10.1016/j.psychres. 2005.09.013

Lamm, C., Batson, C. D., and Decety, J. (2007). The neural substrate of human empathy: effects of perspective-taking and cognitive appraisal. J. Cogn. Neurosci. 19, 42-58. doi: 10.1162/jocn.2007. 19.1.42

Lamm, C., Decety, J., and Singer, T. (2011). Meta-analytic evidence for common and distinct neural networks associated with directly experienced pain and empathy for pain. Neuroimage 54, 2492-2502. doi: 10.1016/j.neuroimage.2010.10.014

Lawrence, E. J., Shaw, P., Giampietro, V. P., Surguladze, S., Brammer, M. J., and David, A. S. (2006). The role of 'shared representations' in social perception and empathy: an fMRI study. Neuroimage 29, 1173-1184. doi: $\quad 10.1016 /$ j.neuroimage. 2005 . 09.001

Legrain, V., Iannetti, G. D., Plaghki, L., and Moureaux, A. (2011). The pain matrix reloaded: a salience detection system for the body. Prog. Neurobiol. 93, 111-124. doi: 10.1016/j.pneurobio.2010.10.005

Lockwood, P. L., Sebastian, C. L., McCrory, E. J., Hyde, Z. H., Gu, X., De Brito, S. A., et al. (2013).
Association of callous traits with reduced neural response to others' pain in children with conduct problems. Curr. Biol. 23, 1-5. doi: 10.1016/j.cub.2013.04.018

Mahmut, M. K., Homewood, J., and Stevenson, R. J. (2008). The characteristics of non-criminals with high psychopathy traits: are they similar to criminal psychopaths. J. Res. Pers. 42, 679-692. doi: 10.1016/j.jrp.2007.09.002

Marsh, A. A., and Blair, R. J. R. (2008). Deficits in facial affect recognition among anti-social populations: a meta-analysis. Neurosci. Biobehav. Rev. 32, 454-465. doi: 10.1016/j.neubiorev.2007.08.003

Marsh, A. A., Finger, E. C., Fowler, K. A., Adalio, C. J., Jurkowitz, I. T., Schechter, J. C., et al. (2013). Empathic responsiveness in amygdala and anterior cingulate cortex in youths with psychopathic traits. J. Child Psychol. Psychiatry 54, 900-910. doi: 10.1111/jcpp.12063

Marsh, A. A., Finger, E. C., Fowler, K. A., Jurkowitz, I. T. N., Schelchter, J. C., Yu, H. H., et al. (2011). Reduced amygdala-orbitofrontal connectivity during moral judgments in youths with disruptive disorders and psychopathic traits. Psychiatry Res. 194, 279-286. doi: 10.1016/j.pscychresns.2011.07.008

Menon, V., and Uddin, L. Q. (2010). Saliency, switching, attention and control: a network model of insula function. Brain Struct. Funct. 214, 655-667. doi: 10.1007/s00429-0100262-0

Miller, P. A., and Jansen op de Haar, M. A. (1997). Emotional, cognitive, behavioral, and temperament characteristics of high-empathy children. Motiv. Emot. 21, 109-125. doi: 10.1023/A:1024430532155

Müller, J., Sommer, M., Wagner, V., Lange, K., Taschler, H., Röder, C. H., et al. (2003). Abnormalities in emotion processing within cortical and subcortical regions in criminal psychopaths: evidence from a functional magnetic resonance imaging study using pictures with emotional content, Biol. Psychol. 54, 152-162. doi: 10.1016/S00063223(02)01749-3

Newman, J. P., and Lorenz, A. R. (2003). "Response modulation and emotion processing: implications for psychopathy and other dysregulatory psychopathology," in Handbook of Affective Sciences, ed Davidson, R. J (New York, NY: Oxford University Press), 904-929.

Nichols, S. R., Svetlova, M., and Brownell, C. A. (2009). The role of social understanding and empathic disposition in young children's responsiveness to ditress in parents and peers. Cogn. Brain Behav. 13, 449-478.

Oswald, P. A. (1996). The effects of cognitive and affective perspective taking on empathic concern and altruistic helping. J. Soc. Psychol. 136, 613-623. doi: 10.1080/002 24545.1996.9714045

Rameson, L. T., Morelli, S. A., and Lieberman, M. D. (2011). The neural correlates of empathy: experience, automaticity, and prosocial behavior. J. Cogn. Neurosci. 24, 235-245. doi: 10.1162/jocn_a_ 00130

Rankin, K. P., Gorno-Tempini, M. L., Allison, S. C., Stanley, C. M., Glenn, S., Weiner, M. W., et al. (2006). Structural anatomy of empathy in neurodegenerative disease. Brain 129, 2945-2956. doi: 10.1093/brain/ awl254

Rankin, K. P., Kramer, J. H., Mychack, P., and Miller, B. L. (2003). Double dissociation of social functioning in frontotemporal dementia. Neurology 60, 266-271. doi: 10.1212/01.WNL.0000041497.0769 4.D2

Roth-Hanania, R., Davidov, M., and Zahn-Waxler, C. (2011). Empathy development from 8 to 16 months: early signs of concern for others. Infant Behav. Dev. 34, 447-458. doi: 10.1016/j.infbeh.2011.04.007

Ruby, P., and Decety, J. (2003). What you believe versus what you think they believe. A neuroimaging study of conceptual perspective taking. Eur. J. Neurosci. 17, 2475-2480. doi: 10.1046/j.1460-9568.2003.02673.x

Ruby, P., and Decety, J. (2004). How would you feel versus how do you think she would feel. A neuroimaging study of perspective taking with social emotions. $J$. Cogn. Neurosci. 16, 988-999. doi: 10.1162/0898929041502661

Salekin, R. T. (2002). Psychopathy and therapeutic pessimism: Clinical lore or clinical reality? Clin. Psychol. Rev. 22, 79-112. doi: 10.1016/S02727358(01)00083-6

Saygin, Z. M., Osher, D. E., Augustinack, J., Fischl, B., and Gabrieli, J. D. E. (2011). Connectivity-based segmentation of human amygdala nuclei using probabilistic tractography. Neuroimage 56, 1353-1361. doi: 10.1016/j.neuroimage.2011.03.006

Schultz, W., Apicella, P., Scarnati, E., and Ljungberg, T. (1992). Neuronal activity in monkey ventral striatum related to the expectation of reward. J. Neurosci. 12, 4595-4610.
Sergerie, K., Chochol, C., and Armony, J. L. (2008). The role of the amygdala in emotional processing: a quantitative meta-analysis of functional neuroimaging studies. Neurosci. Biobehav. Rev. 32, 811-830. doi: 10.1016/j.neubiorev.2007.12.002

Shamay-Tsoory, S., Harari, H., AharonPeretz, J., and Levkovitz, Y. (2010). The role of the orbitofrontal cortex in affective theory of mind deficits in criminal offenders with psychopathic tendencies. Cortex 46, 668-677. doi: 10.1016/j.cortex.2009. 04.008

Shamay-Tsoory, S. G. (2009). "Empathic processing: its cognitive and affective dimensions and neuroanatomical basis," in The Social Neuroscience of Empathy, eds J. Decety and W. Ickes (Cambridge: MIT press), 215-232.

Shamay-Tsoory, S. G., Tibi-Elhanany, Y., and Aharon-Peretz, J. (2006). The ventromedial prefrontal cortex is involved in understanding affective but not cognitive theory of mind stories. Soc. Neurosci. 1, 149-166. doi: 10.1080/174709106 00985589

Shamay-Tsoory, S. G., Tomer, R., Berger, B. D., and Aharon-Peretz, J. (2003). Characterization of empathy deficits following prefrontal brain damage: the role of the right ventromedial prefrontal cortex. J. Cogn. Neurosci. 15, 324-337. doi: 10.1162/089892903321593063

Singer, T., and Decety, J. (2011). "Social neuroscience of empathy," in The Oxford Handbook of Social Neuroscience, eds J. Decety and J. T. Cacioppo (New York, NY: Oxford University Press), 551-564. doi: 10. 1093/oxfordhb/9780195342161.013. 0037

Singer, T., and Lamm, C. (2009). Social neuroscience of empathy. Ann. N.Y. Acad. Sci. 1156, 81-96. doi 10.1111/j.1749-6632.2009.04418.x

Szalavitz, M., and Perry, B. D. (2010). Born for Love. New York, NY: Harper Collins.

Underwood, B., and Moore, B. (1982). Perspective-taking and altruism. Psychol. Bull. 91, 143-173. doi: 10.1037/0033-2909.91.1.143

Van Honk, J., and Schutter, D. J. L. G. (2006). Unmasking feigned sanity: a neurobiological model of emotion processing in primary psychopathy. Cogn. Neuropsychiatry 11, 285-306. doi: 10.1080/13546800500233728

Van Lange, P. A. M. (2008). Does empathy triggers only altruistic motivation: how about selflessness and justice. Emotion 8, 766-774. doi: 10.1037/a0013967 
Valentini, E. (2010). The role of anterior insula and anterior cingulate in empathy for pain. J. Neurophysiol. 104, 584-585. doi: 10.1152/jn.00487.2010

Vollm, B. A., Taylor, A. N. W., Richardson, P., Corcoran, R., Stirling, J., McKie, S., et al. (2006). Neural correlates of theory of mind and empathy: a functional magnetic resonance imaging study in a nonverbal task. Neuroimage 29, 90-98. doi: 10.1016/j.neuroimage. 2005.07.022

Wager, T. D., and Nichols, T. E. (2003). Optimization of experimental design in fMRI: a general framework using a genetic algorithm. Neuroimage 18, 293-309. doi: 10.1016/S1053-8119(02)000460

Young, L., and Dungan, J. (2012). Where in the brain is morality. Everywhere and maybe nowhere. Soc. Neurosci. 7, 1-10. doi: 10.1080/ 17470919.2011.569146

Zaki, J., and Ochsner, K. (2012). "The cognitive neuroscience of sharing and understanding others' emotions," in Empathy: from Bench to Bedside, ed J. Decety (Cambridge: MIT press), 207-226.

Zaki, J., Ochsner, K. N., Hanelin, J., Wager, T. D., and Mackey, S. C. (2007). Different circuits for different pain: patterns of functional connectivity reveal distinct networks for processing pain in self and others. Soc. Neurosci. 2, 276-291. doi: 10.1080/1747091070 1401973

Conflict of Interest Statement: The authors declare that the research was conducted in the absence of any commercial or financial relationships that could be construed as a potential conflict of interest.

Received: 16 July 2013; accepted: 01 August 2013; published online: 24 September 2013.

Citation: Decety J, Chen C, Harenski C and Kiehl KA (2013) An fMRI study of affective perspective taking in individuals with psychopathy: imagining another in pain does not evoke empathy. Front. Hum. Neurosci. 7:489. doi: 10.3389/ fnhum.2013.00489

This article was submitted to the journal Frontiers in Human Neuroscience. Copyright (C) 2013 Decety, Chen, Harenski and Kiehl. This is an openaccess article distributed under the terms of the Creative Commons Attribution License (CC BY). The use, distribution or reproduction in other forums is permitted, provided the original author(s) or licensor are credited and that the original publication in this journal is cited, in accordance with accepted academic practice. No use, distribution or reproduction is permitted which does not comply with these terms. 